\title{
Actinic Superficial Folliculitis after Sun Exposure in a 29-Year-0ld Woman
}

\author{
Rute Lopes Caçola1, Pedro Caiano Gil² \\ ${ }^{1}$ Internal Medicine Department, Hospital Pedro Hispano-Unidade Local de Saúde de Matosinhos, Matosinhos, \\ Portugal \\ ${ }^{2}$ Internal Medicine Departament, Centro Hospitalar de Vila Nova de Gaia e Espinho, Vila Nova de Gaia, Portugal \\ Email: rutelopescacola@gmail.com
}

Received 4 March 2016; accepted 11 April 2016; published 14 April 2016

Copyright (C) 2016 by authors and Scientific Research Publishing Inc.

This work is licensed under the Creative Commons Attribution International License (CC BY). http://creativecommons.org/licenses/by/4.0/

(c) (i) Open Access

\section{Abstract}

The authors report the clinical case of a 29-year-old Caucasian woman who presented with clinicopathological findings and a previous outbreak all suggestive of actinic superficial folliculitis, a rarely reported and probably misdiagnosed phototoxic sun-induced dermatosis first described by Nieboer in 1985. Despite the exuberance of this cutaneous eruption, it is usually auto-limited, reinforcing the importance of its knowledge, for eviction of unnecessary diagnostic tests and therapies. Mechanisms of pathogenesis postulated include ultraviolet A radiation and local heat. This photodermatosis presents as monomorphic, superficial, pustular, and non-pruritic folliculitis affecting the upper body but not the face, usually arising on neck, back, shoulders and upper trunk. The follicular pustules emerge $24-72 \mathrm{~h}$ after intense exposure to heat and/or sunlight and fade spontaneously in 5 - 10 days, without scarring. This patient showed a 48-hour latency period; the number of pustules and area of the body affected were proportional to the duration of the sunlight exposure; the eruption lasted approximately 10 days. Actinic superficial folliculitis has a specific histology with follicular subcorneal sterile pustules and a mixed inflammatory infiltrate around hair follicles, probably secondary to keratinocytes and Langerhans cells involvement in the immunomodulatory actions of ultraviolet radiation. Recurrence under identical conditions may occur, after a latency period of at least 4 weeks, but usually about 1 year. Actinic superficial folliculitis and related follicular conditions are probably underdiagnosed and subsequently there is insufficient scientific information available to clinicians. Being familiar with these entities is of the utmost importance, since it can be crucial for their management.

\section{Keywords}

Actinic Superficial Folliculitis, Subcorneal Sterile Pustules, Sun Exposure, Photodermatosis 


\section{Introduction}

Pustules are circumscribed collections of white blood cells and serous fluid. Many skin lesions will present with papules or vesicles (clear fluid); those presenting with pustules occur less frequently. It is important to recognize the morphological pattern of pustules because it may imply a different spectrum of differential diagnosis as well as treatment. While the differential diagnosis of pustules is broad, several defining features can aid in narrowing down the possibilities in an efficient manner: the patient's age and general health, the distribution and the duration of the lesions. Many follicular conditions associated with exposure to intense sunlight or heat or with immune alterations have been described, namely actinic superficial folliculitis (ASF) [1]-[4], actinic folliculitis (AF) [5]-[7], acne aestivalis (AA) [8] [9], disseminate and recurrent infundibulofolliculitis (RIF) [10]-[12], steroid acne (SA) [13] [14] and cytotoxic folliculitis in acute graft-versus-host disease (CFGvHD) [15] [16]. These conditions share some clinical and histological similarities, as discussed below [3].

ASF is a rare phototoxic sun-induced dermatosis first described by Nieboer in 1985. Mechanisms of pathogenesis postulated include ultraviolet A (UVA) radiation and local heat [1] [2]. UVA radiation may predispose to inflammatory reactions in the hair follicle infundibulum, by an immune or irritative mechanism. This photodermatosis presents as monomorphic, superficial, pustular, and non-pruritic folliculitis affecting the upper body but not the face, usually arising on neck, back, shoulders and upper trunk. The follicular pustules emerge 24 - 72 hours after intense exposure to heat and/or sunlight and fade spontaneously in 5 - 10 days, without scarring [2]-[4]. ASF has a specific histology with follicular subcorneal sterile pustules and a mixed inflammatory infiltrate around hair follicles, probably secondary to keratinocytes and Langerhans cells involvement in the immunomodulatory actions of UV radiation. Recurrence under identical conditions may occur, after a latency period of at least 4 weeks, but usually about 1 year [3] [4].

The authors report the clinical case of a 29-year-old Caucasian woman who presented with clinicopathological findings and a previous outbreak all suggestive of ASF, a rarely reported and probably misdiagnosed acute photodermatosis. Despite the exuberance of this cutaneous eruption, it is usually a self-limited disease, reinforcing the importance of its knowledge, for eviction of unnecessary diagnostic tests and therapies [2]-[4].

\section{Case Presentation}

A 29-year-old Caucasian woman presented to the Emergency Department with an extensive cutaneous follicular pustulosis with surrounding erythema distributed over her neck, shoulders, upper trunk (Figure 1(a) and Figure 1(b)) and back (Figure 2). The face, arms and lower body were spared. Areas of the chest not exposed to the sun remained also free of lesions. The pustular lesions were non-pruritic, painless and aroused within 2 days of that year's first sun exposure, even with the use of sunscreen. The number of pustules and area of the body affected were proportional to the duration of the sunlight exposure.

There were no other symptoms or significant epidemiological context. No medications or cosmetics had been used.

The patient had reported the same symptoms one year before, with less extensive follicular pustules emerging three days after her first sunbath in Summer. The pustules were also localized on her back, chest and shoulders, sparing the face and the areas of skin covered by the bra. Her general condition was then unaffected and the cutaneous eruption resolved spontaneously within 7 days. Her medical history was negative for common acne and other diseases and she had no relevant family history.

On the admission she was apyretic and hemodynamically stable and, besides the cutaneous eruption, physical examination was otherwise unremarkable.

Laboratory evaluation demonstrated elevated inflammatory markers (leukocyte count 15,130/ $\mu \mathrm{L}$ with 85\% neutrophils, C-reactive protein $110 \mathrm{mg} / \mathrm{L}$ ). Hemoglobin, platelet count, ionogram, liver, renal and thyroid function tests, serum immunoglobulins IgA, IgM and IgG, serum protein electrophoresis and immunological study (complement, rheumatoid factor, antinuclear, anti-double-stranded DNA, antineutrophilcytoplasmatic, antiSjögren's syndrome A/B and anti-thyroid antibodies) were normal. Arterial blood gasometry and urinalysis were also normal. Human Immunodeficiency Virus 1 and 2, Herpes simplex virus 1 and 2 (HSV 1 and 2), VaricellaZoster virus (VZV) and Hepatitis C virus serologies were all negative (IgM and IgG). She was immune to Hepatitis B virus. VDRL and Antistreptolysin-O titer were negative. Chest X-ray was normal.

A skin biopsy was performed on the admission after dermatology internal consultation and subsequently histology revealed subcorneal sterile pustules with mixed inflammatory infiltrate with lymphocytes around hair 


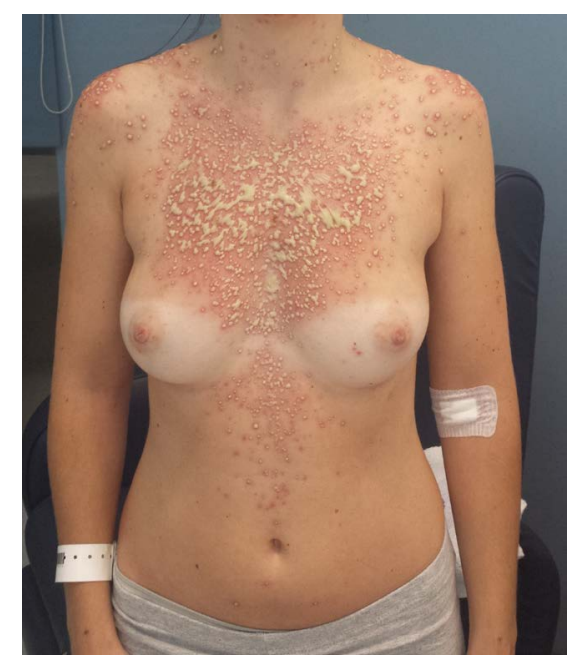

(a)

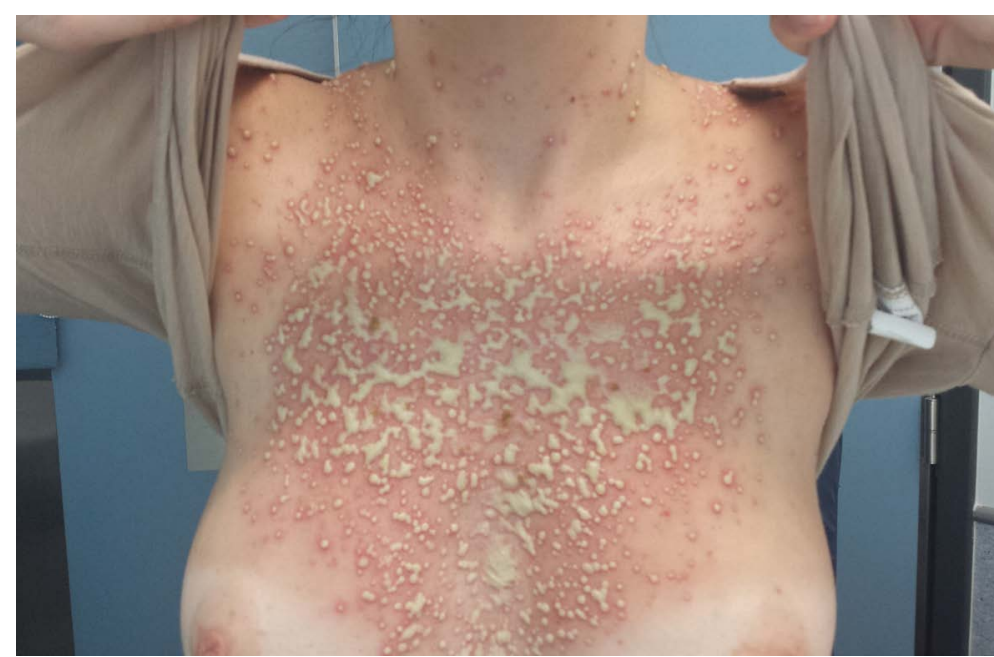

(b)

Figure 1. (a) Monomorfic pustular folliculitis suggestive of actinic superficial folliculitis (neck, shoulders and upper trunk, saving the areas covered by her strapless bra); (b) Closer view of the eruption over the chest, showing some confluent pustules.

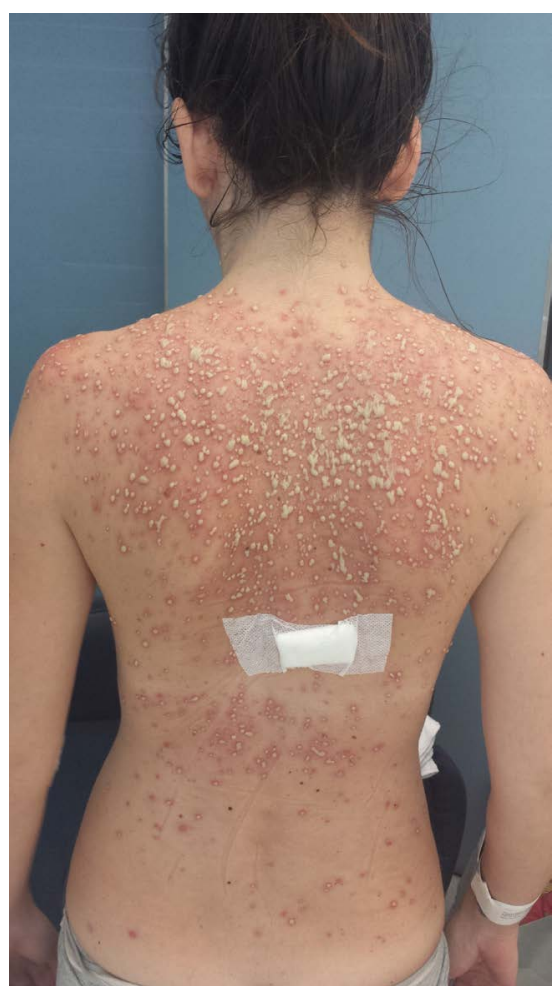

Figure 2. Monomorfic pustular folliculitis suggestive of actinic superficial folliculitis over the back.

follicles infundibulum and sebaceous glands. Bacterial cultures were negative, rulling out Staphylococcus aureus folliculitis. Fungi were not identified on periodic acid-Schiff staining and cultures. Polymerase Chain Reaction of HSV and VZV in biopsy were negative. Blood cultures were also negative.

Empirical therapy with topical Fusidic Acid and intravenous Flucloxacillin 1 gm q6h was started on admission, before definite results of skin biopsy bacterial cultures, assuming the possibility of an infectious folliculitis 
due to gram-positive organisms (including Beta-lactamase-producing Staphylococci). She remained persistently apyretic and hemodynamically stable. Flucloxacillin was stopped on the fourth day of treatment because there was no significant clinical or analytical improvement, skin biopsy histology performed on admission revealed subcorneal sterile pustules and no bacteria or fungi could be cultivated from the pustules.

The eruption lasted approximately 10 days and resolved without further treatment, leaving no scar. Inflammatory markers progressively normalized. Provocative phototest was not performed, but the typical distribution of pustules and the saving of the areas covered by her strapless bra reinforced the pathogenic importance of actinic radiation.

Given the past medical history, clinical features of the cutaneous eruption and the biopsy result, the most likely diagnosis was actinic superficial folliculitis.

The patient was followed in Dermatology and Internal Medicine consultation after hospital discharge. She had no recurrence of the skin rash during one year of follow-up.

\section{Discussion}

The characteristics of this case allowed the authors to rull out superficial pustular folliculitis (whether of infectious origin, or due to other factors such as cosmetics or occlusion), AF, AA, RIF and SA.

Superficial pustular folliculitis (infectious) is not induced by UVA light; skin biopsy histology of this patient revealed subcorneal sterile pustules and no bacteria or fungi could be cultivated from the pustules [2]. There was no history of preceding cosmetic use.

AF, AA, RIF and SA are follicular conditions associated with exposure to intense sunlight or heat or with immune alterations, showing some clinical and histological similarities with ASF. This entities affect the hair follicle only, preferentially the upper follicular epithelium. The follicular lesions present as papules (AF, AA, RIF, SA), pustules (AF, ASF) or papulopustules (RIF) [3]. Time to onset and fade of follicular lesions are also different. In AF, the lesions may arise on face, shoulders, upper trunk and upper arms; the onset usually occurs 4 to 24 hours after UVA light exposure and fade in days to months [3] [6] [7]. AA may onset 15 to 30 days after intense exposure to sun and fade in 3 to 6 months [3] [8] [9]. RIF usually presents from Spring to Autumn with pruritic follicular papules, rarely papulopustules, on neck, trunk, upper arms, buttocks, and proximal thighs; it usually fades in one to several months [3] [10]-[12]. SA may arise 10 to 14 days after corticosteroid therapy on shoulders, upper trunk, upper arms and face and fade in one to several months [3] [13] [14]. Histologically, none of these entities involves comedo formation, at least in the initial phases; infundibular spongiosis and/or necrosis is the most frequent initial finding [3].

There are few case reports detailing ASF. ASF was first described in 1985 by Nieboer, reporting two patients with a recurrent, monomorphic, superficial pustular folliculitis arising on the shoulders, upper trunk and upper extremities, 24 - 36 hours after exposure to sunlight [1]. A short time later, Verbov described two similar patients with a recurrent eruption of monomorphic follicular lesions presenting as pustules of the shoulders, arms and trunk, during warm periods [5]. Labandeira et al. (1997) described the case of a 31-year-old man with sterile follicular pustules on the shoulders, trunk and arms, recurring every year within 48 - 72 hours of the year's first exposure to the sun, with clinicopathological findings closely corresponding to those of ASF as described by Nieboer [3]. Jaeger et al. (2002) reported on a 30-year-old man who presented with an extensive superficial follicular pustulosis on his back, shoulders and upper chest after exposure to intense heat and subsequent sweating on a sunny day; the pustules arose within 24 - 36 hours afterwards and resolved within 10 days without treatment and without scarring [4]. La Berge et al. (2012) reported the case of a 29-year-old man who presented with a 5-year history of an intermittent follicular rash on his back and chest, occurring only when he was exposed to the sun, with his shirt off, 24 to 36 hours after the first sun exposure of the year and resolving spontaneously after 5 to 7 days. This was the first case in which provocative photo testing was done [2].

This case report provides further evidence about this unusual disease.

\section{Conclusion}

ASF and the described related follicular conditions are probably under diagnosed and subsequently there is insufficient scientific information available to clinicians. Being familiar with these entities is of the utmost importance, since it can be crucial for their management. The evolution of this case was, as expected, a highly favourable one, with complete resolution of clinical manifestations. 


\section{Conflict of Interests}

The authors declare that there is no conflict of interests regarding the publication of this paper.

\section{Consent}

Written informed consent was obtained from the patient for publication of this Case report. A copy of the written consent is available for review by the Editor of this journal.

\section{References}

[1] Nieboer, C. (1985) Actinic Superficial Folliculitis; a New Entity? British Journal of Dermatology, 112, 603-606. http://dx.doi.org/10.1111/j.1365-2133.1985.tb15271.x

[2] LaBerge, L., Glassman, S. and Kanigsberg, N. (2012) Actinic Superficial Folliculitis in a 29-Year-Old Man. Journal of Cutaneous Medicine and Surgery, 16, 191-193.

[3] Labandeira, J., Suarez-Campos, A. and Toribio, J. (1998) Actinic Superficial Folliculitis. British Journal of Dermatology, 138, 1070-1074. http://dx.doi.org/10.1046/j.1365-2133.1998.02283.x

[4] Jaeger, C., Hartschuh, W. and Jappe, U. (2003) Actinic Superficial Folliculitis. Journal of The European Academy of Dermatology and Venereology, 17, 562-565. http://dx.doi.org/10.1046/j.1468-3083.2003.00816.x

[5] Verbov, J. (1985) Actinic Folliculitis. British Journal of Dermatology, 113, 630-631. http://dx.doi.org/10.1111/j.1365-2133.1985.tb02392.x

[6] Norris, P.G. and Hawk, J.L.M. (1989) Actinic Folliculitis-Response to Isotretinoin. Clinical and Experimental Dermatology, 14, 69-71. http://dx.doi.org/10.1111/j.1365-2230.1989.tb00889.x

[7] Veysey, E.C. and George, S. (2005) Actinic Folliculitis. Clinical and Experimental Dermatology, 30, 659-661. http://dx.doi.org/10.1111/j.1365-2230.2005.01899.x

[8] Hjorth, N., Sjolin, K.E., Sylvest, B. and Thomsen, K. (1972) Acne Aestivalis-Mallorca Acne. Acta Dermato-Venereologica (Stockh), 52, 61-63.

[9] Mills, O.H. and Kligman, A.M. (1975) Acne Aestivalis. Archives of Dermatology, 111, 891-892. http://dx.doi.org/10.1001/archderm.1975.01630190081008

[10] Owen, W.R. and Wood, C. (1979) Disseminate and Recurrent Infundibulofolliculitis. Archives of Dermatology, 115, 174-175. http://dx.doi.org/10.1001/archderm.1979.04010020020007

[11] Hitch, J.M. and Lund, H.Z. (1968) Disseminate and Recurrent Infundibulofolliculitis: Report of a Case. Archives of Dermatology, 97, 432-435. http://dx.doi.org/10.1001/archderm.1968.01610100072012

[12] Thew, M.A. and Wood, M.G. (1969) Disseminate and Recurrent Infundibulofolliculitis: Report of a Second Case. Archives of Dermatology, 100, 728-733. http://dx.doi.org/10.1001/archderm.1969.01610300078013

[13] Kaidbey, K.H. and Kligman, A.M. (1974) The Pathogenesis of Topical Steroid Acne. Journal of Investigative Dermatology, 62, 31-36. http://dx.doi.org/10.1111/1523-1747.ep12676716

[14] Hurwitz, R.M. (1989) Steroid Acne. Journal of the American Academy of Dermatology, 21, 1179-1181. http://dx.doi.org/10.1016/S0190-9622(89)70325-X

[15] Friedman, K.J., LeBoit, P.E. and Farmer, E.R. (1988) Acute Follicular Graft-vs-Host Reaction. Archives of Dermatology, 124, 688-691. http://dx.doi.org/10.1001/archderm.1988.01670050032014

[16] Murphy, G.F., Lavker, R.M., Whitaker, D. and Korngold, R. (1990) Cytotoxic Folliculitis in GvHD. Evidence of Follicular Stem Cell Injury and Recovery. Journal of Cutaneous Pathology, 18, 309-314.

http://dx.doi.org/10.1111/j.1600-0560.1991.tb01541.x 